\title{
S100A8 Expression May Have a Prognostic Value in CCRCC Reflecting TNM Staging and Fuhrman Nuclear Grade
}

\author{
HYO JUNG AN ${ }^{1,2 *}$, HYUN MIN KOH ${ }^{*}$ and DAE HYUN SONG ${ }^{1,3,4}$ \\ ${ }^{1}$ Department of Pathology, Gyeongsang National University Changwon Hospital, Changwon, Republic of Korea; \\ ${ }^{2}$ Department of Medicine, Gyeongsang National University College of Medicine, Jinju, Republic of Korea; \\ ${ }^{3}$ Gyeongsang National University School of Medicine, Jinju, Republic of Korea; \\ ${ }^{4}$ Gyeongsang Institute of Health Science, Jinju, Republic of Korea
}

\begin{abstract}
Background/Aim: S100A8 is a chemoattractant known to be associated with metastatic niche formation. Herein, we evaluated the prognostic value of S100A8 in patients with clear cell renal cell carcinoma (CCRCC). Materials and Methods: A total of 152 CCRCC patients who have undergone nephrectomy were enrolled. The expression of S100A8 was assessed immunohistochemically using tissue microarray (TMA) blocks of CCRCC. Using statistical analysis, the relationship between S100A8 expression and clinicopathological factors was evaluated. Results: Among 152 TMA cores, 21 (6.9\%) showed higher S100A8 expression. S100A8 expression was significantly increased in cores of patients with higher $T$ stage $(\geq 2, p<0.001)$ and higher Fuhrman nuclear grade $(\geq 3, p<0.001)$. Multivariate analysis confirmed that high expression of S100A8 was significantly correlated to poor disease-free survival (hazard ratio, 2.601; 95\% confidence interval (CI), 1.020-6.628; pvalue $=0.045)$. Conclusion: S100A8 expression may have a prognostic value in CCRCC reflecting TNM staging and Fuhrman nuclear grade.
\end{abstract}

Renal cell carcinoma constitutes one of the most frequently diagnosed cancers, owing to the advanced abdominal imaging technique. They are often incidentally detected as small tumors, however, locally aggressive cancers with distant metastasis have been identified at diagnosis in up to $17 \%$ of cases worldwide. During the last decade, the treatment of metastatic renal cell carcinoma has been developed through

*Hyo Jung An and Hyun Min Koh have contributed equally to this article as first Authors.

Correspondence to: Dae Hyun Song, MD, Department of Pathology, Gyeongsang National University School of Medicine, 79 Gangnam-ro, Jinju-si, Gyeongsangnam-do, Republic of Korea. Tel: +82 552143150, Fax: +82 552143174, e-mail: golgy@ hanmail.net

Key Words: Clear cell, prognosis, renal cell carcinoma, S100A8, tumor progression. discovering specific targets in molecular pathways. Even though many molecular markers have been investigated for the prognosis of renal cell carcinoma, none of them has yet to enhance the most prevalent system used, the TNM classification. S100A8 is one of the chemo-attractants known to be associated with the metastatic niche in the lungs before cancer metastasizes to the target organ (1). They accumulate in bone marrow-derived hematopoietic cells (HPCs) and indirectly attract them to pre-metastatic lungs, inducing an inflammation-like state $(1,2)$. Over time they accelerate the migration of primary tumor cells to the metastatic niche in lung tissue (3). Meanwhile, there is evidence that S100A8 takes part in cancer cell migration and invasion in metastatic organs in cases such as hepatic cancer (4), prostate cancer (5), breast cancer (6), and malignant melanoma (7). Since S100A8 data regarding renal cancer are scarce, in this investigation, we used TMA blocks and statistical analysis to evaluate the impact of S100A8 in clear cell renal cell carcinoma (CCRCC).

\section{Materials and Methods}

Patients and clinicopathological factors. We collected tumor samples from 152 patients who had undergone surgical resection for CCRCC at the Gyeongsang National University Hospital (Jinju, Korea) between January 2000 and December 2009. These specimens were staged using the eighth edition of the American Joint Committee on Cancer (AJCC) TNM Classification of Malignant Tumors (8). We obtained clinical information by reviewing electronic medical charts. Disease-free survival (DFS) was defined as the duration from the date of surgery to the date of cancer relapse, and disease-specific survival (DSS) was defined as the duration from the date of surgery to the date of death, which was mostly due to CCRCC. This study was approved by the Institutional Review Board of Gyeongsang National University Hospital (2018-07-005) and was conducted in accordance with the principles embodied in the Declaration of Helsinki and its later amendments or comparable ethical standards.

Tissue microarray construction and immunohistochemical staining. Two 2-mm-sized cores were made from each of the 152 cases of the 
representative formalin-fixed, paraffin-embedded blocks. Each core was obtained from the invasive front and the most predominant component including highest grade. Immunohistochemistry was performed by an automated immunostainer (Benchmark Ultra, Ventana Medical Systems Inc., Tucson, AZ, USA) with monoclonal anti-S100A8 antibody at a 1:500 dilution (EPR3554, Abcam, Cambridge, UK). Antigen retrieval was also performed. Lymphoid cells from the tonsils were used as a positive control. Capillary endothelial cells were used as an internal control since there have been previous reports about S100A8 and A9 co-localized in the vascular endothelium where leukocytes migrate into tissue-inflamed areas. The intensity of the staining in the nucleus of tumor cells was graded as follows: tumor cells that had a weaker staining intensity than capillary endothelial cells were considered to have low S100A8 expression, and the remaining tumor cells were considered to have high S100A8 expression. The representative images are shown in Figure $1 \mathrm{~A}$ and $\mathrm{B}$.

Statistical analysis. The relationship of S100A8 expression with clinicopathological factors was assessed using Pearson's chi-square test. The prognostic impact of S100A8 was analyzed by univariate and multivariate tests by using the Cox proportional hazard model. DFS and DSS were represented by the Kaplan-Meier survival curve. A $p$-value of less than 0.05 was regarded as statistically significant. The analyses were performed using IBM SPSS ver. 24.0 (IBM Corp., Armonk, NY, USA).

\section{Results}

Clinicopathological factors of the patients. The clinicopathological factors of the 152 CCRCC patients are summarized in Table I. The mean age of the patients was 59.9 years (range $=32-83$ years). Amongst them, $109(71.7 \%)$ were men. The distribution of $\mathrm{T}$ stages was as follows: 1a: 91 (59.9\%), 1b: 24 (15.8\%), 2a: 9 (5.9\%), 2b: 3 (2.0\%), 3a: 2 $(1.3 \%), 4: 2$ (1.3\%). With respect to Fuhrman nuclear grade, $26(17.1 \%)$ were grade $1,102(67.1 \%)$ were grade 2,19 $(12.5 \%)$ were grade 3 , and $5(3.3 \%)$ were grade 4 . The mean follow-up period was 4.33 years (range $=0-12.35$ years).

Correlation between S100A8 expression and clinicopathological factors. Immunohistochemical staining for S100A8 revealed high expression in 21 cores $(6.9 \%)$ of CCRCC. S100A8 expression was significantly increased in samples with a higher $\mathrm{T}$ stage $(\geq 2, p<0.001)$ and a higher Fuhrman nuclear grade ( $\geq 3, p<0.001)$, compared to those with a lower stage and a lower nuclear grade (Table II). Patient age $(p=0.151)$ and sex $(p=0.325)$ were not related to S100A8 expression (Table II).

Survival analysis. Univariate analysis revealed that patient age $(p=0.004), \mathrm{T}$ stage $(p<0.001)$, Fuhrman nuclear grade $(p<0.001)$, and S100A8 expression $(p<0.001)$ were associated with DFS and DSS. In multivariate analysis, high S100A8 expression was proven to be an independent prognostic marker in CCRCC. High expression of S100A8 was
Table I. Clinicopathological factors of the clear cell renal cell carcinoma patients.

\begin{tabular}{lc}
\hline Variable & Value $(\%)(\mathrm{n}=152)$ \\
\hline Age, mean (range) & $59.9(32-83)$ \\
Gender (Male/Female) & $109 / 43$ \\
Follow up period, mean (years) & 4.33 \\
T stage & \\
1a & $91(59.9 \%)$ \\
$1 \mathrm{~b}$ & $24(15.8 \%)$ \\
$2 \mathrm{a}$ & $9(5.9 \%)$ \\
$2 \mathrm{~b}$ & $3(2.0 \%)$ \\
$3 \mathrm{a}$ & $21(13.8 \%)$ \\
$3 \mathrm{~b}$ & $2(1.3 \%)$ \\
4 & $2(1.3 \%)$ \\
Fuhrman's nuclear grade & \\
1 & $26(17.1 \%)$ \\
2 & $102(67.1 \%)$ \\
3 & $19(12.5 \%)$ \\
4 & $5(3.3 \%)$ \\
Advanced RCC & \\
Lung metastasis & 9 \\
Multiple metastasis & 6 \\
Bone metastasis & 4 \\
Brain metastasis & 2 \\
Liver metastasis & 1 \\
Local recurrence & 3 \\
Total & 152 patients \\
\hline
\end{tabular}

Values are presented as numbers (\%).

Table II. Correlation between S100A8 expression and clinicopathological factors ( $n=303$ cores).

\begin{tabular}{lrrr}
\hline \multirow{2}{*}{$\begin{array}{l}\text { Clinicopathological } \\
\text { information }\end{array}$} & \multicolumn{2}{c}{ S100A8 expression } & \\
\cline { 2 - 2 } & Low expression & High expression & $p$-Value \\
\hline Age & & & 0.151 \\
$\quad<59$ & $126(95.5)$ & $6(4.5)$ & \\
$\quad \geq 59$ & $156(91.2)$ & $15(8.8)$ & \\
Gender & & & 0.325 \\
$\quad$ Male & $200(92.2)$ & $17(7.8)$ & \\
$\quad$ Female & $82(95.3)$ & $4(4.7)$ & \\
T stage & & & $<0.001$ \\
$\quad<2$ & $223(97.4)$ & $6(2.6)$ & \\
$\geq 2$ & $59(79.7)$ & $15(20.3)$ & \\
Fuhrman nuclear grade & & & $<0.001$ \\
$\quad<3$ & $244(96.1)$ & $10(3.9)$ & \\
$\quad \geq 3$ & $38(77.6)$ & $11(22.4)$ & \\
\hline
\end{tabular}

Values are presented as numbers (\%).

significantly related to poor DFS [hazard ratio $(\mathrm{HR})=2.601$; $95 \%$ confidence interval $(C I)=1.020-6.628 ; p$-value $=0.045]$, but not to DSS in CCRCC (Table III). In addition, KaplanMeier survival curves showed that the group with high 


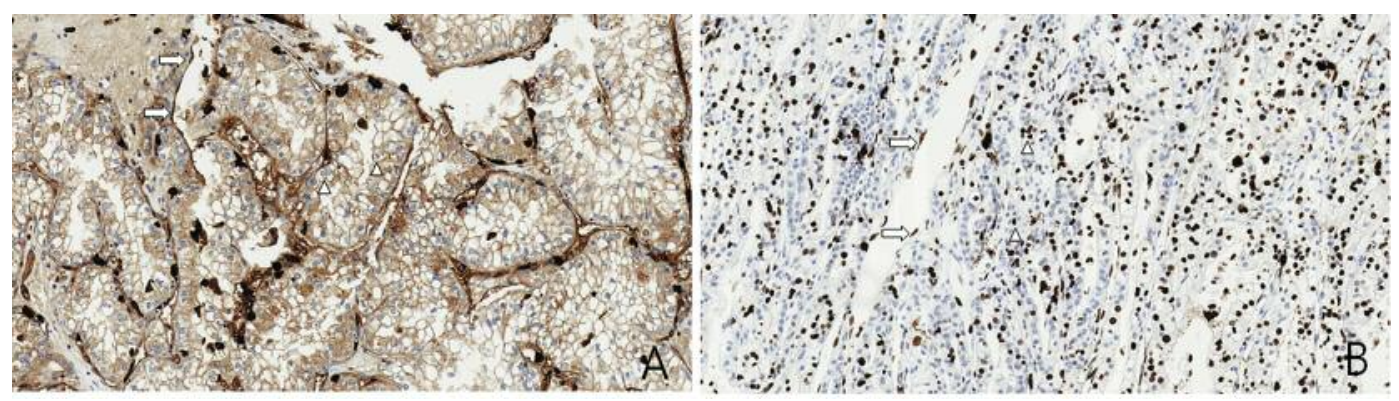

Figure 1. Images of S100A8 staining in clear cell renal cell carcinoma. Tumor cells reveal weaker (A, low expression) or stronger expression (B, high expression) compared to the capillary endothelial cells (arrow, endothelial cells; arrow head, tumor cells; original magnification: 200x).

Table III. Univariate and multivariate analysis of disease-free and disease-specific survival in patients with CCRCC

\begin{tabular}{|c|c|c|c|c|c|c|c|c|}
\hline \multirow[b]{3}{*}{ Variables } & \multicolumn{4}{|c|}{ Univariate analysis } & \multicolumn{4}{|c|}{ Multivariate analysis } \\
\hline & \multicolumn{2}{|l|}{ DFS } & \multicolumn{2}{|l|}{ DSS } & \multicolumn{2}{|c|}{ DFS } & \multicolumn{2}{|l|}{ DSS } \\
\hline & HR $(95 \% \mathrm{CI})$ & $p$-Value & HR $(95 \% \mathrm{CI})$ & $p$-Value & HR $(95 \% \mathrm{CI})$ & $p$-Value & HR $(95 \% \mathrm{CI})$ & $p$-Value \\
\hline Age & & & & & & & & \\
\hline$(<59$ vs. $\geq 59)$ & $3.740(1.538-9.093)$ & 0.004 & $1.762(0.880-3.529)$ & 0.110 & $2.525(1.010-6.313)$ & 0.048 & & \\
\hline $\begin{array}{l}\text { Gender } \\
\text { (male vs. female) }\end{array}$ & $0.423(0.148-1.210)$ & 0.109 & $1.045(0.472-2.311)$ & 0.914 & & & & \\
\hline $\begin{array}{l}\text { T stage } \\
\quad(<2 \text { vs. } \geq 2)\end{array}$ & $21.216(8.688-51.811)$ & $<0.001$ & $30.111(10.628-85.308)$ & $<0.001$ & $17.889(6.629-48.274)$ & $<0.001$ & $21.714(7.472-63.102)$ & $<0.001$ \\
\hline $\begin{array}{l}\text { Fuhrman nuclear grade } \\
\text { ( }<3 \mathrm{vs} . \geq 3)\end{array}$ & $6.300(3.100-12.804)$ & $<0.001$ & $6.286(3.265-12.103)$ & $<0.001$ & $4.100(1.671-10.060)$ & 0.002 & $3.416(1.621-7.196)$ & 0.001 \\
\hline $\begin{array}{l}\text { S100A8 expression } \\
\text { (low vs. high) }\end{array}$ & $11.203(5.113-24.547)$ & $<0.001$ & $9.790(4.811-19.920)$ & $<0.001$ & $2.601(1.020-6.628)$ & 0.045 & $1.849(0.825-4.144)$ & 0.135 \\
\hline
\end{tabular}

CCRCC: Clear cell renal cell carcinoma; CI: confidence interval; DFS: disease-free survival; DSS: disease-specific survival; HR: hazard ratio. Bold values denote statistical significance at the $p<0.05$ level.

S100A8 expression was significantly related to poor DFS $(p<0.001)$ and DSS $(p<0.001)$ as compared to the low S100A8 expression group (Figure 2A and B).

\section{Discussion}

The S100 protein was discovered by Moore et al. (1965). To find proteins unique to the nervous system they chromatographed proteins extracted from the brain and liver and then performed gel electrophoresis, where only one protein band that was absent in the liver sample was found to be present in the brain sample (9). As this protein was soluble in a solution of saturated ammonium sulfate, it was called S100 (9). Intracellularly, S100 proteins are involved in cell proliferation, differentiation, migration, invasion, $\mathrm{Ca}^{2+}$ signaling, and inflammation (10). They interact with various target proteins, including enzymes, receptors, transcription factors, and nucleic acids (10). Among the 24 members of the S100 protein family (10), S100A8 and S100A9 are chemoattractants indirectly produced by distant primary tumors (3). They attract HPCs to induce an inflammationlike state in the lungs called the "pre-metastatic niche". Along with factors such as TNF-alpha, matrix metalloproteinase (MMP)-9, and TGF-beta, which are secreted by HPCs, metastatic tumor cells engraft the niche to facilitate micrometastases. The engagement of endothelial progenitor cells (EPCs) at this metastatic site initiates angiogenesis, thus leading to macrometastases (2).

There is evidence that S100A8 and S100A9 play roles in cancer cell migration and invasion of metastatic organs in cases such as melanoma (7), and cancers of the liver (3), prostate (11), and breast (6). In melanoma, extracellular S100A8 and S100A9 contribute to tumor progression and metastasis. Melanoma-derived exosomes promote metastatic niche formation by educating bone marrow (BM)-derived progenitor cells. Peinado et al. (2012) found that these exosomes with pre-educated BM cells accelerate tumor growth (7). On performing gene expression profiling of the 

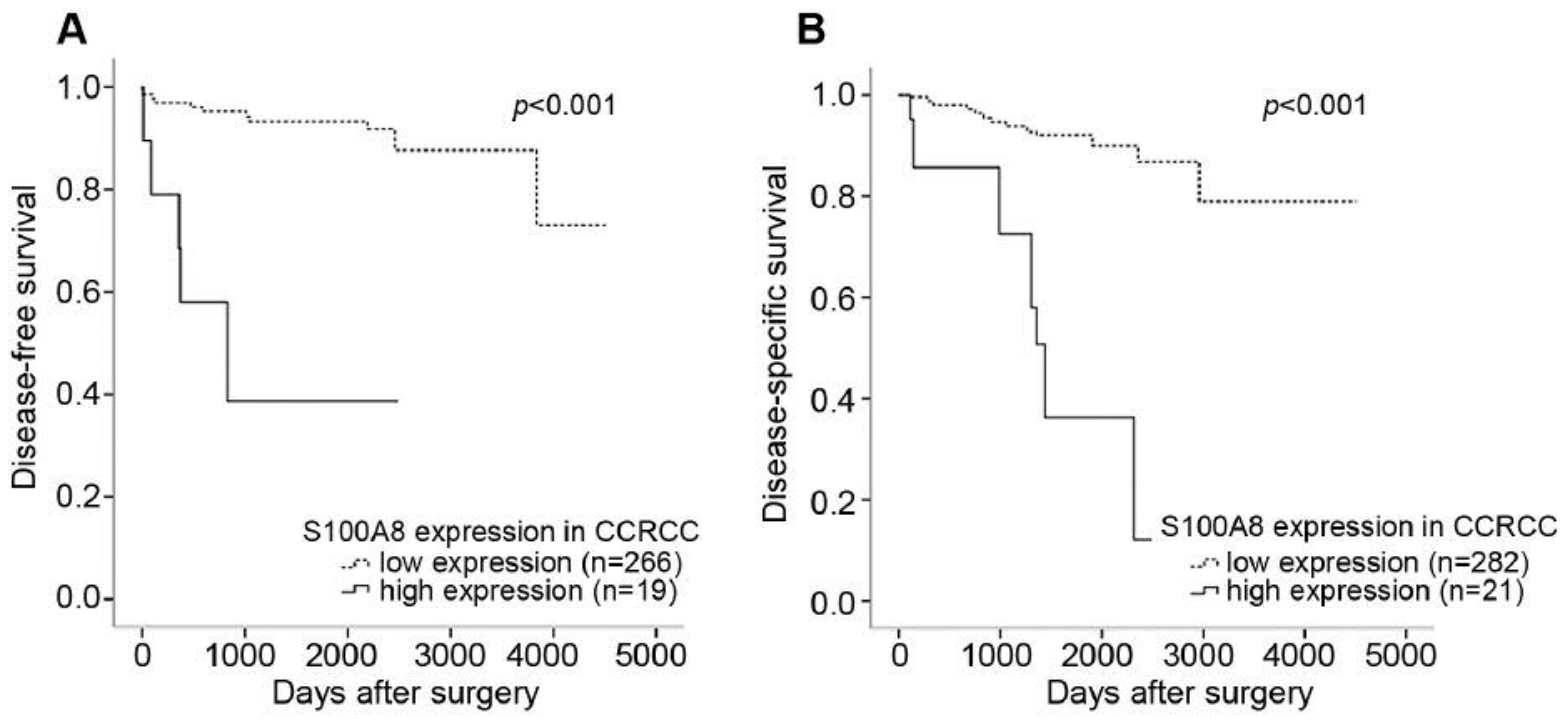

Figure 2. Kaplan-Meier survival curve between groups with high and low S100A8 expression in clear cell renal cell carcinoma. S100A8-high expression group revealed significantly poor disease-free survival (A) and disease-specific survival (B) compared to the S100A8-low expression group.

pre-metastatic niche lung tissue, 130 unique genes related to extracellular matrix remodeling and inflammation were found, which included genes encoding heat-shock proteins, S100A8, and S100A9, all of which are related to early metastatic events (7). Similarly, S100A8 induced the expression of serum amyloid A3 (SAA3) in the premetastatic niche (lung) but not in the metastatic target organ (liver). SAA3 in the pre-metastatic phase upregulated NF-kB signaling in TLR4-mediated cascade and increased liver metastasis (3).

Meanwhile, Hermani et al. (2005) observed that S100A8 and S100A9 showed increased expression in the invasive front of the prostate, potentially due to their secretion and stimulation of MMP expression in the stroma (11). Moon et al. (2008) proved that the knockdown of S100A8 and S100A9 expression significantly reduced H-Ras-mediated cell invasion and MMP-2/MMP-9 induction in human breast cancer cells (6). Since MMPs play an important part in tumor invasion and matrix remodeling, they confirmed the functional significance of S100A8 and S100A9 in inducing the invasive phenotype of human breast epithelial cells (6). Mirza et al. suggested that high levels of S100A8 expression were one of the intrinsic molecular mechanisms which contribute to the progression of kidney cancer. Using molecular docking studies, they proved that the S100A8 protein is a potential target for therapeutic intervention in kidney cancer (12). Finally, Zhang et al. revealed that differential expression of S100A8/A9 exists in four groups including, renal cyst, renal hamartoma, normal kidney, and renal cell carcinoma. They examined differential expression among different types of cancer cell populations through bioinformatics analysis, however, failed to reflect the differential grades of RCC, maybe due to the small sample sizes (13).

To the best of our knowledge, this is the first study to suggest that $\mathrm{S} 100 \mathrm{~A} 8$ is related to the nuclear grade and advanced stage in CCRCC. S100A8 expression was significantly increased in samples of patients with a higher T stage $(\geq 2, p<0.001)$ and a higher Fuhrman's nuclear grade $(\geq 3, p<0.001)$. Additionally, CCRCC with a higher S100A8 expression demonstrated unfavorable DFS even in the multivariate analysis $\mathrm{HR}=2.601 ; 95 \% \mathrm{CI}=1.020-6.628$; $p$-value $=0.045)$. According to the guidelines of the AJCC (8), tumor sizes 4,7 , and 10 centimeters are important criteria to divide the pathological $\mathrm{T}$ stages into $1 \mathrm{a}, 1 \mathrm{~b}, 2 \mathrm{a}$, and $2 \mathrm{~b}$. Tumors extending to major blood vessels are classified as stage 3(a-c). Stage 4 comprises tumors that invade areas beyond Gerota's fascia or the ipsilateral adrenal gland. Through our observation, S100A8 is not only relevant to the pre-metastatic phase, as previously discovered in several studies, but has also been proven to be related to tumor progression and metastasis in CCRCC. As such, S100A8 might be a prognostic factor in CCRCC. Future studies focusing on the interactions between S100A8 and other receptors or cytokines that initiate the metastatic process in CCRCC are required for better insight.

In this investigation, we used TMA blocks and statistical analysis to evaluate the predictive role of S100A8 in tumor progression of CCRCC. Therapies targeting the immuneregulatory functions of $\mathrm{S} 100 \mathrm{~A} 8$ and S100A9 for the regulation of tumor growth and metastasis could be useful. In conclusion, S100A8 may have prognostic value in CCRCC, reflecting TNM staging or Fuhrman nuclear grade. 


\section{Conflicts of Interest}

The Authors declare that they have no conflicts of interest regarding this study.

\section{Authors' Contributions}

HJ An: Project development, Data analysis, Manuscript writing; HM Koh: Manuscript writing, Data management; DH Song: Manuscript editing, Data analysis, Supervisor.

\section{References}

1 Hiratsuka S, Watanabe A, Aburatani $\mathrm{H}$ and Maru Y: Tumourmediated upregulation of chemoattractants and recruitment of myeloid cells predetermines lung metastasis. Nat Cell Biol 8(12): 1369, 2006. PMID: 17128264. DOI: $10.1038 / \mathrm{ncb} 1507$

2 Psaila B and Lyden D: The metastatic niche: adapting the foreign soil. Nat Rev Cancer 9(4): 285, 2009. PMID: 19308068. DOI: $10.1038 / \mathrm{nrc} 2621$

3 Hiratsuka S, Watanabe A, Sakurai Y, Akashi-Takamura S, Ishibashi S, Miyake K, Shibuya M, Akira S, Aburatani $\mathrm{H}$ and Maru Y: The S100A8-serum amyloid A3-TLR4 paracrine cascade establishes a pre-metastatic phase. Nat Cell Biol 10(11): 1349, 2008. PMID: 18820689. DOI: $10.1038 /$ ncb1794

4 Lim SY, Yuzhalin AE, Gordon-Weeks AN and Muschel RJ: Tumor-infiltrating monocytes/macrophages promote tumor invasion and migration by upregulating S100A8 and S100A9 expression in cancer cells. Oncogene 35(44): 5735, 2016. PMID: 27086923. DOI: $10.1038 /$ onc.2016.107

5 Miao L, Grebhardt S, Shi J, Peipe I, Zhang J and Mayer D: Prostaglandin E2 stimulates S100A8 expression by activating protein kinase A and CCAAT/enhancer-binding-protein-beta in prostate cancer cells. Int J Biochem Cell Biol 44(11): 1919-1928, 2012. PMID: 22728165. DOI: 10.1016/j.biocel.2012.06.015

6 Moon A, Yong HY, Song JI, Cukovic D, Salagrama S, Kaplan D, Putt D, Kim H, Dombkowski A and Kim HR: Global gene expression profiling unveils S100A8/A9 as candidate markers in H-ras-mediated human breast epithelial cell invasion. Mol Cancer Res 6(10): 1544-1553, 2008. PMID: 18922970. DOI: 10.1158/1541-7786.MCR-08-0189
7 Peinado H, Alečković M, Lavotshkin S, Matei I, Costa-Silva B, Moreno-Bueno G, Hergueta-Redondo M, Williams C, GarcíaSantos G, Ghajar C, Nitadori-Hoshino A, Hoffman C, Badal K, Garcia BA, Callahan MK, Yuan J, Martins VR, Skog J, Kaplan RN, Brady MS, Wolchok JD, Chapman PB, Kang Y, Bromberg $\mathrm{J}$ and Lyden D: Melanoma exosomes educate bone marrow progenitor cells toward a pro-metastatic phenotype through MET. Nat Med 18(6): 883, 2012. PMID: 22635005. DOI: $10.1038 / \mathrm{nm} .2753$

8 Amin MB, Edge SB and Greene FL: AJCC (American Joint Committee on Cancer) Cancer Staging Manual, 8th edition. Amin MB, Edge SB and Greene FL (eds.). Chicago, Springer, 2017.

9 Moore BW: A soluble protein characteristic of the nervous system. Biochem Biophys Res Commun 19(6): 739-744,1965. PMID: 4953930. DOI: 10.1016/0006-291x(65)90320-7

10 Donato R, Cannon BR, Sorci G, Riuzzi F, Hsu K, Weber DJ and Geczy CL: Functions of S100 proteins. Curr Mol Med 13(1): 2457, 2013. PMID: 22834835.

11 Hermani A, Hess J, De Servi B, Medunjanin S, Grobholz R, Trojan L, Angel $\mathrm{P}$ and Mayer D: Calcium-binding proteins S100A8 and S100A9 as novel diagnostic markers in human prostate cancer. Clin Cancer Res 11(14): 5146-5152, 2005. PMID: 16033829. DOI: 10.1158/1078-0432.CCR-05-0352

12 Mirza Z, Schulten HJ, Farsi HM, Al-Maghrabi JA, Gari MA, Chaudhary AG, Abuzenadah AM, Al-Qahtani MH and Karim S: Impact of S100A8 expression on kidney cancer progression and molecular docking studies for kidney cancer therapeutics. Anticancer Res 34(4): 1873-1884, 2014. PMID: 24692722.

13 Zhang L, Jiang H, Xu G, Wen H, Gu B, Liu J, Mao S, Na R, Jing Y, Ding Q and Zhang Y: Proteins S100A8 and S100A9 are potential biomarkers for renal cell carcinoma in the early stages: results from a proteomic study integrated with bioinformatics analysis. Molecular medicine reports 11(6): 4093-4100, 2015. PMID: 25673070. DOI: 10.3892/mmr.2015.3321

Received July 31, 2019

Revised August 19, 2019

Accepted August 20, 2019 